\title{
The Voice of a Psychiatry Resident Doctor During COVID-19 Outbreak in Mumbai, India
}

Pooja Kapri,, Pawan Gadgile. ${ }^{1}$

\section{The Experience}

As the world has been brought to a standstill with the recent COVID-19 outbreak declared a pandemic on 11th March 2020, we both junior resident doctors in the Department of Psychiatry, Lokmanya Tilak Municipal Medical College and General Hospital, Mumbai, India would like to share our experiences in this situation.

The first recorded case of COVID-19 infection in India was on 3oth January 2020 and with the rising cases since then the Government of India issued a nationwide lockdown, starting 22nd March 2020 and further extending it to 30th April 2020. The current statistics of total open cases in India have surpassed 9000 as of 14 thApril 2020. The decree limits the movement of general public for procurement of essential goods and services while our frontline warriors, like doctors, nurses and paramedical staff, essential goods' manufacturers and distributors, police forces and sanitation workers have been tirelessly at work.

Being resident doctors, we have been in the center of this pandemic. We have seen an unprecedented increase in number of anxious patients developing panic disorder and patients going into substance withdrawal in the past few weeks. Many people developed what is called Corona anxiety and they feel that they may contract the virus. Mild fever and cough were thought of as COVID and anxiety developed. Excessive protection and obsessiveness about social distancing and protection developed in people.1

A number of patients on maintenance medications of psychiatric illnesses have been having difficulty in reaching out to the doctors for prescriptions as a result of the lockdown, and have had acute exacerbation of their complaints. Telemedicine has been proposed as the need of the hour, although highly debatable. The Ministry of Health at Family Welfare, India has recently released guidelines to be followed.

There is no stringent body that would monitor the quality of these services and whether ethical standards are adhered to. It is important that professionals and agencies offering telepsychiatry services have professionals who are qualified and trained in this regard to some extent.2

Even compliant patients that came to our Out Patient Department had sleep and appetite disturbances, easy irritability, breakdowns over trivial issues and anxiousness. We also received innumerable queries and concerns in the recent days from relatives and friends having difficulties in managing their stress, culminating into emotional outbursts. Lending an open ear, simple reassurances and general directives for prevention against COVID infection as well promoting mental well-being and self-care has been the way forward for us. We have emphasized that social distancing should not mean emotional distancing, and in these trying times we need to stay connected with our family, friends and loved ones via electronic means.

The medical and paramedical fraternity have been under tremendous stress themselves with the mounting number of daily new cases and deaths, long and tiring work hours (14-18 hours a day), scarcity of hospital beds, lack of treatment protocols and Personal Protective Equipment (PPE), constant worry about contracting the infection and taking it home; thus putting our families at risk, staying in the hospital premises for days together; away from our own family has all been taking a toll on our mental health as well.

Good mental health care for these doctors, nurses and ancillary staff is very important and this will help in developing a stable healthcare infrastructure to combat COVID. There is also a need for training these staff in communication skills and building their resilience for the tough times that they shall face ahead.3

Many psychiatrists have felt that the current pandemic has made psychiatry appear as a non-essential field. Hospitals and many centers have allowed that their non-essential staff to stay home which include basic sciences and psychiatry in some quarters but the question of essentiality and non-essential is a personal one that we have to answer. We cannot shy away as we are doctors first and psychiatrists later. Consultation-liaison psychiatrists work closely with the medical and surgical teams and also know about the worries that their colleagues undergo. Consultation-liaison psychiatrists usually traverse through the hospital and multiple specialties system and will be on the frontline in this pandemic.4 While we use telepsychiatry to manage our patients, it is vital that we realize we are as important on the frontline as we are on a video screen or telephone call.

India has been facing one of the biggest crises of recent times both socially and economically, putting a physical, mental and emotional strain on patients, general public and healthcare providers alike. The World Health Organization has praised India's commitment in combating and containing the pandemic, setting an example for others to follow. Only time will tell about the final outcome, as we give in our best fight against COVID-19 while providing help, support and empathy to fellow beings.

1. Psychiatry Resident, Lokmanya Tilak Municipal medical college and General Hospital, Mumbai, India.

About the Author: Dr Pooja Kapri is currently Second year resident doctor in Department of Psychiatry, Lokmanya Tilak Municipal Medical College and General Hospital, Mumbai, India of a three-year degree program. 


\section{Experience}

\section{References}

1. Sood S. Psychological effects of the Coronavirus disease-2019 pandemic. Res Hum Med Educ 2020;7(1):23-26.

2. Qiu J, Shen B, Zhao M, Wang Z, Xie B, Xu Y. A nationwide survey of psychological distress among Chinese people in the COVID-19 epidemic: implications and policy recommendations. Gen Psychiatr. 2020 Mar 6;33(2):e100213.
3. Adams JG, Walls RM. Supporting the health care workforce during the COVID-19 global epidemic. JAMA. 2020 Mar 12. [Epub ahead of print]

4. Duan L, Zhu G. Psychological interventions for people affected by the COVID-19 epidemic. Lancet Psychiatry. 2020 Apr;7(4):300-302.

\section{Acknowledgments}

None

Conflict of Interest Statement at Funding

The Authors have no funding, financial relationships or conflicts of interest to disclose.

Author Contributions

Conceptualization, Methodology, Writing - Original Draft, Writing - Review đt Editing, Visualization: PK and PG.

Cite as:

Kapri P, Gadgile P. The Voice of a Psychiatry Resident Doctor During COVID-19 Outbreak in Mumbai, India. Int J Med Students. 2020 Jan Apr;8(1):73-74.

This work is licensed under a Creative Commons Attribution 4.0 International License

ISSN 2076-6327

This journal is published by the University Library System, University of Pittsburgh as part of the Digital Publishing Program and is co-sponsored by the University of Pittsburgh Press. 